\title{
新型三嗪类席夫碱大环化合物的合成与表征
}

\author{
李小安花成文* 苟小锋赵军龙陈 邦 \\ (西北大学化学与材料科学学院 合成与天然功能分子化学教育部重点实验室 西安 710069)
}

\begin{abstract}
摘要 以三聚氯氭、二乙胺、香草醛、邻苯二胺、对苯二胺、联苯胺、4,4'-二氨基-二苯醚、4,4'-二氨基-二苯甲烷为原 料, 经取代、成环反应得到了一类新型的含三嗪环的大环席夫碱化合物，并通过 IR, ${ }^{1} \mathrm{H}$ NMR, ESI/MS 和元素分析对其 结构进行了表征. 其紫外研究结果显示, 席夫碱大环 $\mathbf{3 b}$ 对 $\mathrm{Cu}^{2+}, \mathbf{3 a}, \mathbf{3 c}$ 对 $\mathrm{Fe}^{3+}$ 有识别作用.
\end{abstract}

关键词 三聚氯氧; 席夫碱; 大环化合物; 合成; 表征

\section{Synthesis and Characterization of Novel Schiff Base Macrocyclic Compounds of 1,3,5-Triazine}

\author{
Li, Xiaoan Hua, Chengwen* Gou, Xiaofeng Zhao, Junlong Chen, Bang \\ (Key Laboratory of Synthetic and Natural Functional Molecule Chemistry (Ministry of Education), \\ College of Chemistry and Materials Science, Northwest University, Xi'an 710069)
}

\begin{abstract}
Several novel Schiff base macrocyclic compounds of 1,3,5-triazine were synthesized from cyanuric chloride, diethylamine, vanillin, $o$-phenylenediamine, $p$-phenylenediamine, benzidine, 4,4'-diamino-ether, 4,4'-diamino-diphenyl methane by substitution and cyclization, and their structures were characterized by ${ }^{1} \mathrm{H}$ NMR, IR, ESI/MS techniques and elemental analysis. The studies on the UV-Vis absorption spectroscopies show that Schiff base $\mathbf{3} \mathbf{b}$ has a selective recognition for $\mathrm{Cu}^{2+}$, and $3 \mathbf{a}, \mathbf{3 c}$ for $\mathrm{Fe}^{3+}$.

Keywords cyanuric chloride; Schiff base; macrocyclic compound; synthesis; characterization
\end{abstract}

大环是一种重要的主-客体化合物, 它们具有独特 的空间构型，在分子识别、模拟酶、功能材料等方面的 应用受到了广泛关注, 新型大环化合物的设计与合成也 成为当代有机化学与超分子化学领域的热点问题 ${ }^{[1]}$. 自 Robson 等 ${ }^{[2]}$ 首次合成席夫碱大环后, 基于其 $\mathrm{N}, \mathrm{O}$ 等杂 原子与金属的配位作用，在超分子化学、催化、医药、 磁性及光学材料等方面得到了深入的研究 ${ }^{[3]}$. 近年来, 席夫碱大环化合物的合成及应用研究异常活跃. 席夫碱 大环的合成方法主要分为三种: (1)直接合成法(高度稀 释法); (2)模板合成法; (3)非模板合成法 ${ }^{[4]}$. 三聚氯氭具 有独特的反应活性, 由它引入的三嗪环具有热氧稳定性 和生物活性, 其既能充当氢键的提供者, 又能充当氢键 的接受者, 在离子识别和模拟酶方面有潜在的功能 ${ }^{[5]}$. 本文以三聚氯氰、二乙胺、香草醛、邻苯二胺、对苯二 胺、联苯胺、4,4'-二氨基-二苯醚、4,4'-二氨基-二苯甲烷 为原料, 采用直接合成法, 以 $[2+2]$ 环合的方式合成了 一类新型的含三嗪环的席夫碱大环化合物, 通过 ${ }^{1} \mathrm{H}$
NMR, IR, ESI/MS 和元素分析对其结构进行了表征. 合 成路线如 Scheme 1.

\section{1 实验部分}

\section{1 仪器与试剂}

XT-4 型显微熔点仪, 温度计未校正; EQUINOX55 傅立叶红外光谱仪; micrOTOF-Q $\|$ ESI/MS 电喷雾质谱 仪; INOVA-400 MHz 型核磁共振仪; 德国 EL-III 元素分 析仪. 溶剂和试剂均为市售分析纯试剂, 除特别注明外, 未经进一步处理.

\section{2 合成}

1.2.1 2-二乙胺基-4,6-二氯-1,3,5-三嗪(1)的合成

$3.68 \mathrm{~g}(0.02 \mathrm{~mol})$ 三聚氯氰和 $2.76 \mathrm{~g}(0.02 \mathrm{~mol})$ 无水 碳酸钾加入到 $40 \mathrm{~mL}$ THF 中, 置于冰浴中搅拌. $1.46 \mathrm{~g}$ $(0.02 \mathrm{~mol})$ 二乙胺用 $20 \mathrm{~mL}$ THF 溶解后, 慢慢滴加入上 述体系. $30 \mathrm{~min}$ 滴完，体系变为浑浊状. 用 TLC 监控反

\footnotetext{
* E-mail: huachengwen@163.com

Received October 31, 2011; revised December 12, 2011; published online January 11, 2012.
} 
<smiles>CCN(CC)CCc1ccc(O)c(OC)c1</smiles><smiles></smiles><smiles>CCN(CC)c1nc(Oc2ccc(C=O)cc2OC)nc(Oc2ccc(C=O)cc2OC)n1</smiles>
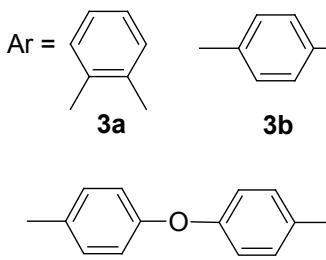

$3 d$

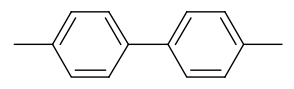

$3 c$

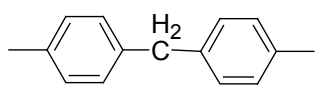

$3 e$

Scheme 1

应进程, 继续反应 $3 \mathrm{~h}$, 停止反应, 将反应液过滤得无色 澄清液体. 将液体倾入 $200 \mathrm{~mL}$ 水中, 静置过夜, 得到无 色晶体状物质. 抽滤后, 烘干, 得到 $3.75 \mathrm{~g} \mathrm{1}$, 产率 85.2\%. m.p. $78 \sim 79{ }^{\circ} \mathrm{C} ;{ }^{1} \mathrm{H}$ NMR $\left(\mathrm{CDCl}_{3}, 400 \mathrm{MHz}\right) \delta$ : 1.22 (t, $J=7.1 \mathrm{~Hz}, 6 \mathrm{H}), 3.63$ (q, $J=7.1 \mathrm{~Hz}, 4 \mathrm{H})$; IR (KBr) v: $2959\left(\mathrm{CH}_{3}\right), 2874\left(\mathrm{CH}_{2}\right), 1586,1475,1379$ (三嗪环的骨 架振动吸收), $1226(\mathrm{C}-\mathrm{N}-\mathrm{C}), 790(\mathrm{C}-\mathrm{Cl}) \mathrm{cm}^{-1}$. Anal. calcd for $\mathrm{C}_{7} \mathrm{H}_{10} \mathrm{Cl}_{2} \mathrm{~N}_{4} \mathrm{C} 38.03, \mathrm{H}$ 4.56, $\mathrm{N}$ 25.34; found $\mathrm{C}$ 38.08, H 4.60, N 25.28.

\subsubsection{2-二乙胺基-4,6-二(2-甲氧基-4-醛基苯氧基)- 1,3,5-三嗪(2)的合成}

在 $250 \mathrm{~mL}$ 单口瓶中加入 $2.2 \mathrm{~g}(0.01 \mathrm{~mol})$ 化合物 $\mathbf{1}$, $3.04 \mathrm{~g}(0.02 \mathrm{~mol})$ 香草醛, $2.76 \mathrm{~g}(0.02 \mathrm{~mol})$ 无水碳酸钾,

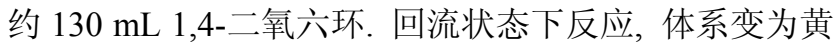
色浑浊状. TLC 监控反应进程, $24 \mathrm{~h}$ 后停止反应, 抽滤得 浅黄色透明滤液, 减压蒸干 1,4-二氧六环得粗产品. 用 无水乙醇重结晶, 得 $3.5 \mathrm{~g}$ 白色晶体状固体 2 , 产率 89.7\%. m.p. $132 \sim 133{ }^{\circ} \mathrm{C} ;{ }^{1} \mathrm{H} \mathrm{NMR}\left(\mathrm{CDCl}_{3}, 400 \mathrm{MHz}\right) \delta$ : 1.02 (t, $J=7.0 \mathrm{~Hz}, 6 \mathrm{H}), 3.38$ (q, $J=7.0 \mathrm{~Hz}, 4 \mathrm{H}), 3.85$ (s, $6 \mathrm{H}), 7.26 \sim 7.28(\mathrm{~m}, 2 \mathrm{H}), 7.30(\mathrm{~s}, 2 \mathrm{H}), 7.45 \sim 7.46(\mathrm{~m}$, 2H), $9.94(\mathrm{~s}, 2 \mathrm{H})$; IR (KBr) v: 2975, $2936\left(\mathrm{CH}_{3}\right), 2828$ $\left(\mathrm{CH}_{2}\right), 1695$ (CHO), 1600, 1500, 1384, 1318(芳环和三嗪 环骨架振动交叠吸收), $1024(\mathrm{C}-\mathrm{O}-\mathrm{C}$ 的伸缩振动 $)$, $812,718 \mathrm{~cm}^{-1}$. Anal. calcd for $\mathrm{C}_{23} \mathrm{H}_{24} \mathrm{~N}_{4} \mathrm{O}_{6} \mathrm{C} 61.05, \mathrm{H}$ 5.35, N 12.38; found C 61.12, H 5.30, N 12.33.

\section{2 .3 新型三嗪类席夫碱大环化合物 $\mathbf{3} \mathbf{a}$ 的合成}

在 $100 \mathrm{~mL}$ 三口瓶中加入 $0.452 \mathrm{~g}(0.001 \mathrm{~mol})$ 化合物 2 和 $20 \mathrm{~mL}$ 无水甲醇, $\mathrm{N}_{2}$ 保护, 回流状态下搅拌. 慢慢滴 加 $0.108 \mathrm{~g}(0.001 \mathrm{~mol})$ 邻苯二胺和 $15 \mathrm{~mL}$ 无水甲醇的溶
液, 历时 $30 \mathrm{~min}$ 滴毕. 滴完后反应液变为黄色浑浊状, TLC 监控反应，继续摚拌 $4 \mathrm{~h}$ 到达反应终点，过滤，用热 甲醇, 热乙醇反复洗涤后烘干, 得到 $0.18 \mathrm{~g}$ 黄色固体 $\mathbf{3 a}$, 产率 34.4\%. m.p. $178 \sim 180{ }^{\circ} \mathrm{C} ;{ }^{1} \mathrm{H}$ NMR $\left(\mathrm{CDCl}_{3}, 400\right.$ MHz) $\delta: 1.23(\mathrm{t}, J=7.3 \mathrm{~Hz}, 12 \mathrm{H}), 3.37$ (q, $J=7.3 \mathrm{~Hz}, 8 \mathrm{H})$, $3.79(\mathrm{~s}, 12 \mathrm{H}), 6.92 \sim 6.94(\mathrm{~m}, 4 \mathrm{H}), 6.97(\mathrm{~s}, 4 \mathrm{H}), 7.17 \sim$ $7.21(\mathrm{~m}, 4 \mathrm{H}), 7.82 \sim 7.84(\mathrm{~m}, 8 \mathrm{H}), 8.38(\mathrm{~s}, 4 \mathrm{H})$; IR $(\mathrm{KBr})$ v: $2968\left(\mathrm{CH}_{3}\right), 2933\left(\mathrm{CH}_{2}\right), 1591(\mathrm{C}=\mathrm{N}), 1500,1458$, 1375(芳环和三嗪环骨架振动交叠吸收), $1205(\mathrm{C}-\mathrm{N}-\mathrm{C}$ 的振动吸收), $1028(\mathrm{C}-\mathrm{O}-\mathrm{C}$ 的伸缩振动), 741(苯环的 邻位取代) $\mathrm{cm}^{-1}$; ESI/MS m/z: $1049.4347(\mathrm{M}+\mathrm{H})^{+}$. Anal. calcd for $\mathrm{C}_{58} \mathrm{H}_{56} \mathrm{~N}_{12} \mathrm{O}_{8} \mathrm{C} 66.40, \mathrm{H} \mathrm{5.38}, \mathrm{N} \mathrm{16.02}$; found $\mathrm{C}$ 66.36, H 5.33, N 16.05.

同法制得席夫碱大环化合物 $\mathbf{3 b}$, 浅黄色粉末，产率 40.2\%. m.p. $>300{ }^{\circ} \mathrm{C} ;{ }^{1} \mathrm{H} \mathrm{NMR}\left(\mathrm{CDCl}_{3}, 400 \mathrm{MHz}\right) \delta: 1.25$ (t, $J=8.0 \mathrm{~Hz}, 12 \mathrm{H}), 3.37$ (q, $J=8.0 \mathrm{~Hz}, 8 \mathrm{H}), 3.69$ (s, 12H), $6.91 \sim 6.93(\mathrm{~m}, 4 \mathrm{H}), 6.99(\mathrm{~s}, 4 \mathrm{H}), 7.11 \sim 7.13(\mathrm{~m}, 4 \mathrm{H})$, $7.31 \sim 7.33(\mathrm{~m}, 8 \mathrm{H}), 8.25(\mathrm{~s}, 4 \mathrm{H})$; IR (KBr) v: 2971, 2935 $\left(\mathrm{CH}_{3}\right), 2870\left(\mathrm{CH}_{2}\right), 1591(\mathrm{C}=\mathrm{N}), 1503,1459,1380$ (芳环 和三嗪环骨架振动交叠吸收 $), 1212(\mathrm{C}-\mathrm{N}-\mathrm{C}$ 的振动吸 收), 1029 ( $\mathrm{C}-\mathrm{O}-\mathrm{C}$ 的伸缩振动), 810 (苯环的对位取代) $\mathrm{cm}^{-1}$; ESI/MS m/z: $1049.4456(\mathrm{M}+\mathrm{H})^{+}$. Anal. calcd for $\mathrm{C}_{58} \mathrm{H}_{56} \mathrm{~N}_{12} \mathrm{O}_{8}$ : C 66.40, H 5.38, N 16.02; found $\mathrm{C} 66.45, \mathrm{H}$ 5.29, N 16.09.

同法制得席夫碱大环化合物 3c，浅黄色粉末，产率 52.1\%. m.p. $>300{ }^{\circ} \mathrm{C} ;{ }^{1} \mathrm{H} \mathrm{NMR}\left(\mathrm{CDCl}_{3}, 400 \mathrm{MHz}\right) \delta: 1.29$ $(\mathrm{t}, J=8.0 \mathrm{~Hz}, 12 \mathrm{H}), 3.41(\mathrm{q}, J=8.0 \mathrm{~Hz}, 8 \mathrm{H}), 3.77(\mathrm{~s}, 12 \mathrm{H})$, $6.96 \sim 7.00(\mathrm{~m}, 4 \mathrm{H}), 7.04(\mathrm{~s}, 4 \mathrm{H}), 7.07 \sim 7.09(\mathrm{~m}, 4 \mathrm{H})$, $7.42 \sim 7.46(\mathrm{~m}, 16 \mathrm{H}), 8.32(\mathrm{~s}, 4 \mathrm{H}) ; \mathrm{IR}(\mathrm{KBr}) v: 2969,2933$ $\left(\mathrm{CH}_{3}\right), 2867\left(\mathrm{CH}_{2}\right), 1588(\mathrm{C}=\mathrm{N}), 1502,1458,1374$ (芳环 
和三嗪环骨架振动交叠吸收), $1211(\mathrm{C}-\mathrm{N}-\mathrm{C}$ 的振动吸 收), $1028(\mathrm{C}-\mathrm{O}-\mathrm{C}$ 的伸缩振动), 856(苯环的对位取代) $\mathrm{cm}^{-1}$; ESI/MS m/z: $1201.5046(\mathrm{M}+\mathrm{H})^{+}$. Anal. calcd for $\mathrm{C}_{70} \mathrm{H}_{64} \mathrm{~N}_{12} \mathrm{O}_{8}$ : C 69.98, H 5.37, N 13.99; found C 69.94, H 5.43, N 13.95 .

同法制得席夫碱大环化合物 3d, 浅黄色粉末, 产率 45.2\%. m.p. $216 \sim 217{ }^{\circ} \mathrm{C} ;{ }^{1} \mathrm{H} \mathrm{NMR}\left(\mathrm{CDCl}_{3}, 400 \mathrm{MHz}\right) \delta$ : $1.28(\mathrm{t}, J=7.5 \mathrm{~Hz}, 12 \mathrm{H}), 3.39$ (q, $J=7.5 \mathrm{~Hz}, 8 \mathrm{H}), 3.74$ (s, $12 \mathrm{H}), 6.85 \sim 6.87(\mathrm{~m}, 4 \mathrm{H}), 6.97(\mathrm{~s}, 4 \mathrm{H}), 7.01 \sim 7.04(\mathrm{~m}$, 4H), $7.44 \sim 7.46(\mathrm{~m}, 16 \mathrm{H}), 8.27(\mathrm{~s}, 4 \mathrm{H})$; IR (KBr) v: 2966, $2931\left(\mathrm{CH}_{3}\right), 2864\left(\mathrm{CH}_{2}\right), 1587(\mathrm{C}=\mathrm{N}), 1497,1458$, 1373(芳环和三嗪环骨架振动交叠吸收), $1207(\mathrm{C}-\mathrm{N}-\mathrm{C}$ 的振动吸收), $1028(\mathrm{C}-\mathrm{O}-\mathrm{C}$ 的伸缩振动), 834, 806(苯 环的对位取代) $\mathrm{cm}^{-1}$; ESI/MS m/z: $1233.4882(\mathrm{M}+\mathrm{H})^{+}$. Anal. calcd for $\mathrm{C}_{70} \mathrm{H}_{64} \mathrm{~N}_{12} \mathrm{O}_{10}$ : C 68.17, H 5.23, N 13.63; found C 68.21, H 5.25, N 13.58.

同法制得大环席夫碱化合物 $\mathbf{3 e}$, 浅黄色粉末，产率 43.6\%. m.p.194 $195{ }^{\circ} \mathrm{C} ;{ }^{1} \mathrm{H}$ NMR $\left(\mathrm{CDCl}_{3}, 400 \mathrm{MHz}\right) \delta$ : $1.26(\mathrm{t}, J=7.5 \mathrm{~Hz}, 12 \mathrm{H}), 3.39(\mathrm{q}, J=7.5 \mathrm{~Hz}, 8 \mathrm{H}), 3.73$ (s, $12 \mathrm{H}), 3.76(\mathrm{~s}, 4 \mathrm{H}), 6.94 \sim 6.96(\mathrm{~m}, 4 \mathrm{H}), 7.14(\mathrm{~s}, 4 \mathrm{H})$, $7.17 \sim 7.19$ (m, 4H), $7.44 \sim 7.46(\mathrm{~m}, 16 \mathrm{H}), 8.27$ (s, 4H); IR (KBr) v: $2967\left(\mathrm{CH}_{3}\right), 2868\left(\mathrm{CH}_{2}\right), 1588(\mathrm{C}=\mathrm{N}), 1500$, 1459，1375(芳环和三嗪环骨架振动交叠吸收), 1208 $(\mathrm{C}-\mathrm{N}-\mathrm{C}$ 的振动吸收 $), 1027(\mathrm{C}-\mathrm{O}-\mathrm{C}$ 的伸缩振动 $)$, 806(苯环的对位取代) $\mathrm{cm}^{-1}$; ESI/MS m/z: 1229.5359 $(\mathrm{M}+\mathrm{H})^{+}$. Anal. calcd for $\mathrm{C}_{72} \mathrm{H}_{68} \mathrm{~N}_{12} \mathrm{O}_{8}$ : C 70.34, H 5.58, N 13.67; found C 70.29, H 5.60, N 13.71.

\section{3 离子识别实验}

分别移取 $1 \mathrm{~mL}$ 受体 $\mathbf{3 a} \sim \mathbf{3 e}$ 的 $\mathrm{CHCl}_{3}$ 溶液 $\left(1 \times 10^{-4}\right.$ $\mathrm{mol} / \mathrm{L})$ 于一系列 $10 \mathrm{~mL}$ 比色管中. 分别加入 $\mathrm{Li}^{+}, \mathrm{Na}^{+}$, $\mathrm{Mg}^{2+}, \mathrm{Cu}^{2+}, \mathrm{Fe}^{3+}$ 金属离子的高氯酸盐的甲醇溶液 $(1 \times$ $\left.10^{-4} \mathrm{~mol} / \mathrm{L}\right) 0.5 \mathrm{~mL}$, 用 $\mathrm{CHCl}_{3}$ 稀释至刻度, 混合均匀后 放置 $6 \mathrm{~h}$, 于 $25{ }^{\circ} \mathrm{C}$ 测其紫外-可见吸收光谱 $\left(\mathrm{CHCl}_{3}\right.$ 作参 比).

\section{2 结果与讨论}

\section{1 元素分析}

元素分析的测量值与理论值吻合，可以推断生成了 大环席夫碱化合物. 但不能确定环合是按 $[1+1]$ 还是 $[2+2]$ 进行反应的，必须再结合其他检测手段加以确定.

\section{2 质谱分析}

从目标化合物 $\mathbf{3 a} \sim \mathbf{3 e}$ 的质谱分析来看,$m / z$ 1049.4347, 1049.4456, 1201.5046, 1233.4882, 1229.5359 分别是它们的 $\mathrm{M}^{+}+1$ 峰, 由此说明环合是按 $[2+2]$ 的方
式完成的.

\section{$2.3 \mathrm{IR}$ 谱分析}

原料中 $1695 \mathrm{~cm}^{-1}$ 处的醛的 $v_{\mathrm{C}=\mathrm{O}}$ 振动峰消失, 在 $1580 \sim 1600 \mathrm{~cm}^{-1}$ 范围内出现了很强的 $v_{\mathrm{C}=\mathrm{N}}$ 伸缩振动峰, 表明醛基已与胺基缩合生成了席夫碱. 在 2930 2970, $2850 \sim 2870 \mathrm{~cm}^{-1}$ 范围内出现了甲基和亚甲基的对称及 不对称伸缩振动, 对应于原料二乙胺上的 $\mathrm{CH}_{2} \mathrm{CH}_{3}$. $1500,1460,1380 \mathrm{~cm}^{-1}$ 附近的出峰是芳环和三嗪环的骨 架振动交叠吸收引起的, $730 \sim 860 \mathrm{~cm}^{-1}$ 范围内的出峰 验证了苯环上的邻位或对位取代.

\section{$2.4{ }^{1} \mathrm{H}$ NMR 谱分析}

在 ${ }^{1} \mathrm{H}$ NMR 谱中，甲基、亚甲基质子的 $\delta$ 均在 1.22 3.79 范围内, 苯环质子的 $\delta$ 在 $6.85 \sim 7.84$ 范围内, $\mathrm{CH}$ 质 子的 $\delta$ 在 8.30 附近.

\section{5 非模板法成环}

文献中合成席夫碱大环一般采取模板法，但是模板 法成环时需要选择性地加入金属离子，而且成环后还要 进行去离子过程. 文献报道的非模板合成法产率很低, 甚至得不到目标产物. 我们在设计分子时，选择的分子 片段的空间构型更利于偶合成环。因此，本实验中，采 用一锅煮的直接合成法，以较高的产率合成了新型的席 夫碱大环，这种方法比模板合成法简单，少了除模板离 子的麻烦.

本文设计席夫碱大环时，选择了空间结构有利于成 环的分子片段，反应中几乎无副产物生成，目标产物在 无水甲醇中直接以固体形式析出，只需用热的溶剂洗涤 即可得纯度较高的产品.

\section{6 对金属离子的识别}

席夫碱大环 $3 \mathbf{a} \sim 3 \mathbf{e}$ 在 $\mathrm{CHCl}_{3}$ 中的紫外-可见光谱数 据测定显示，它们的紫外最大吸收 $\lambda_{\text {max }}$ 分别为 302, 356, $351,313,305 \mathrm{~nm}$. 大环分别与 $\mathrm{Li}^{+}, \mathrm{Na}^{+}, \mathrm{Mg}^{2+}, \mathrm{Cu}^{2+}$, $\mathrm{Fe}^{3+}$ 等离子作用后, $\mathrm{Cu}^{2+}$ 使 $\mathbf{3 b}$ 的 $\lambda_{\text {max }}$ 红移至 $452 \mathrm{~nm}$ (图 1b). $\mathrm{Fe}^{3+}$ 使 $\mathbf{3 a}$ 的 $\lambda_{\text {max }}$ 红移至 $305 \mathrm{~nm}$ (图 1a), $\mathbf{3 c}$ 的 $\lambda_{\text {max }}$ 红 移至 $506 \mathrm{~nm}$ (图 1c), 其它作用后无明显变化. 由此结果 表明，席夫碱大环 $\mathbf{3 b}$ 对 $\mathrm{Cu}^{2+}, \mathbf{3 a}, \mathbf{3} \mathbf{c}$ 对 $\mathrm{Fe}^{3+}$ 有选择识别 作用.

席夫碱大环由于其自身的 $\pi$ 体系空腔，能与很多金 属形成配合物，而对金属离子具有一定的识别作用. 本 文合成的席夫碱大环的空腔大小与某些过渡金属离子 相匹配，可以形成配合物. 且席夫碱大环存在多个 $\mathrm{C}=$ $\mathrm{N}$ 官能团和 $\mathrm{N}, \mathrm{O}$ 杂原子, $\mathrm{Fe}^{3+}, \mathrm{Cu}^{2+}$ 与 $\mathrm{N}, \mathrm{O}$ 原子有很 强的配位能力，便于其进行络合，进而使其紫外吸收发 生红移. 离子识别实验表明, $\mathbf{3 a}, \mathbf{3} \mathbf{c}$ 与 $\mathrm{Fe}^{3+}, \mathbf{3 b}$ 与 $\mathrm{Cu}^{2+}$ 更容易发生包结络合，形成配合物。 

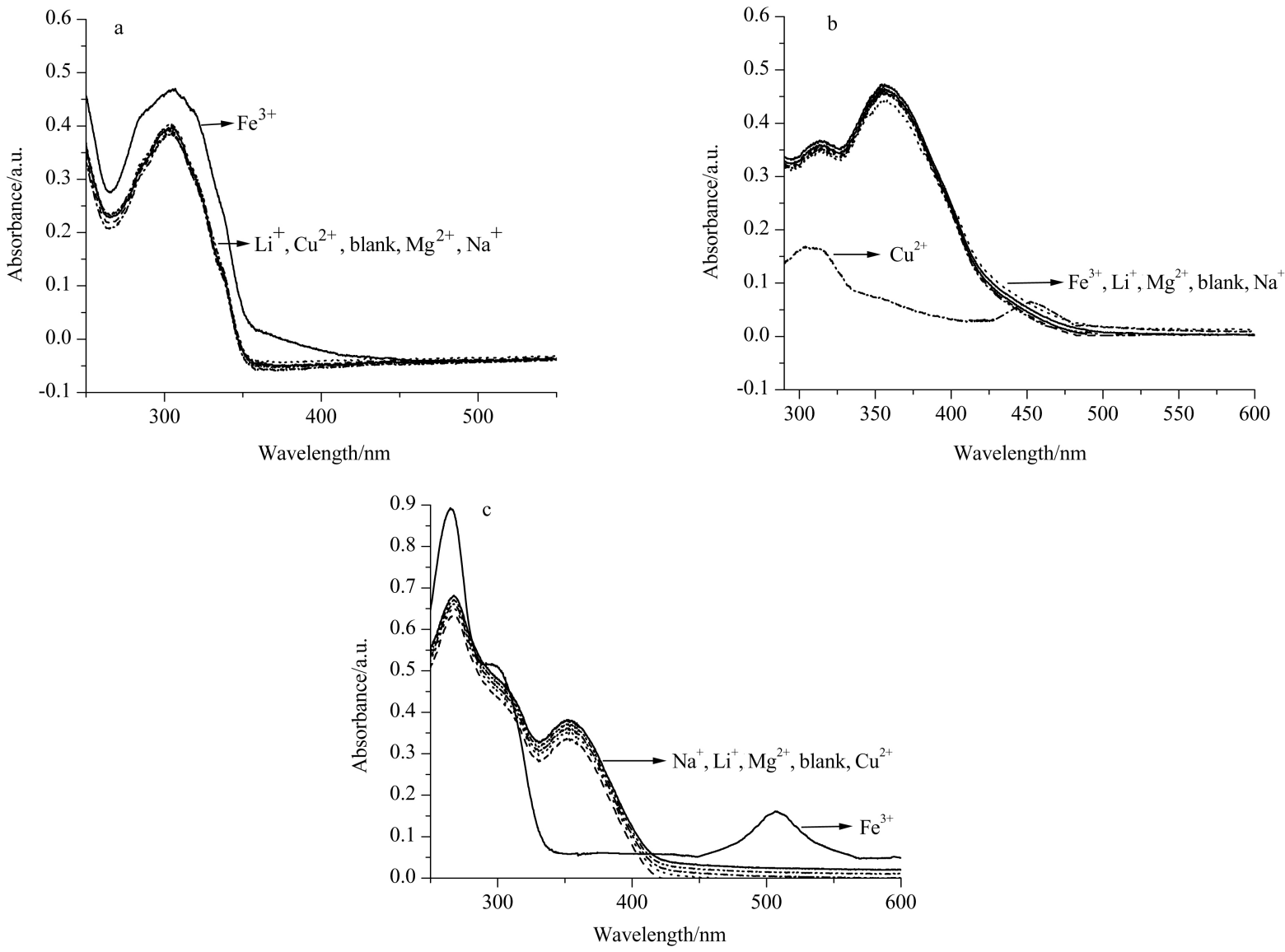

图 1 金属离子存在时受体分子 $3 \mathrm{a}, \mathbf{3 b}, \mathbf{3 c}$ 的紫外吸收光谱

Figure 1 UV-vis absorption spectra of $\mathbf{3 a}, \mathbf{3} \mathbf{b}, \mathbf{3} \mathbf{c}$ in the presence of various metal ions

\section{3 结论}

本文采用一锅煮的直接合成法，以[2+2]环合的方 式, 合成了一类新型的席夫碱大环化合物, 并对它们进 行了表征. 紫外测试结果显示, 席夫碱大环 $\mathbf{3 b}$ 对 $\mathrm{Cu}^{2+}$, 3a、3c 对 $\mathrm{Fe}^{3+}$ 有识别作用.

\section{References}

[1] (a) Daniel, G. R.; Ludger, A. W. J. Am. Chem. Soc. 2009, 131(10), 3721 .

(b) Terence, K. M. L.; Zhu, N. Y.; Vivian, W. W. Y. J. Am. Chem. Soc. 2010, 132, 17646.

(c) Jaeduk, Y.; Youngmee, K.; Sung, J. K.; Chang, H. L. Chem. Commun. 2010, 46, 5449.

[2] Pilkington, N. H.; Robson, R. Aust. J. Chem. 1970, 23, 2225.

[3] (a) Ma, C. T. L.; MacLachlan, M. J. Angew. Chem., Int. Ed. 2005, 44, 4178.

(b) Zheng, X. L.; Jones, C. W.; Weck, M. J. Am. Chem. Soc. 2007, $129,1105$.

(c) Yuan, Z.-L.; Hu, Q.-H.; Wu, Q.; Zhang, M.-Q.; Zhu, B.-X. Chin. J. Org. Chem. 2009, 29, 279 (in Chinese).

(袁泽利, 胡庆红, 吴庆, 张铭钦, 朱必学, 有机化学, 2009, 29,
282.)

(d) Song, Y.-M.; Zhang, Y.-M.; Ma, X.-X.; Zhu, Z.-L.; Xu, J.-P.; Liu, J.-W. Acta Chim. Sinica 2011, 69, 1347 (in Chinese). (宋玉民, 张玉梅, 马新贤, 朱早龙, 许军鹏, 刘景旺, 化学学报, 2011, 69, 1347.)

(e) Leung, D. H.; Bergman, R. G.; Raymond, K. N. J. Am. Chem. Soc. 2007, 129, 2746.

[4] (a) Guerrierop, V. P. A.; Fenton, D, E. Acta Chem. Scand. 1992, 46, 1025.

(b) Busch, D. H.; Vance, A. L.; Kolchinski, A. G. In Comprehensive Supramolecular Chemistry, Pergamon, New York, 1996, Vol. 9, pp. $1 \sim 42$.

(c) Leeland, J. W.; White, F. J.; Love, J. B. J. Am. Chem. Soc. 2011, 133,7320

(d) Martinez, A.; Hemmert, C.; Loup, C.; Barré, G.; Meunier, B. J. Org. Chem. 2006, 71, 1449.

[5] (a) Marta, E. A.; Gloria, M.; Nino, R.; Emilia, S. Chem. Commun. 2010, 46, 5894.

(b) Löwik, D. W. P.; Lowe, C. R. J. Org. Chem. 2001, 2825.

(c) Wang, M. X.; Yang, H. B. J. Am. Chem. Soc. 2004, 126, 15412.

(d) Lv, G.-H.; Hua, C.-W.; Gou, X.-F.; Liu, Y.-F. Chin. J. Org. Chem. 2010, 30, 745 (in Chinese).

(吕国华, 花成文, 荷小锋, 刘艳风, 有机化学, 2010, 30, 745.)

(Li, L.) 